\title{
Frequency of deep convective clouds in the tropical zone from 10 years of AIRS data
}

\author{
H. H. Aumann and A. Ruzmaikin \\ Jet Propulsion Laboratory/California Institute of Technology, Pasadena, CA, USA \\ Correspondence to: H. H. Aumann (aumann@jpl.nasa.gov) \\ Received: 2 February 2013 - Published in Atmos. Chem. Phys. Discuss.: 17 April 2013 \\ Revised: 19 July 2013 - Accepted: 4 October 2013 - Published: 7 November 2013
}

\begin{abstract}
Deep convective clouds (DCCs) have been widely studied because of their association with heavy precipitation and severe weather events. Changes in the properties of DCCs are likely in a changing climate. Ten years of data collected by Atmospheric Infrared Sounder (AIRS) allow us to identify decadal trends in frequency of occurrence of DCCs over land and ocean. In the past, DCCs have been identified in the thermal infrared by three methods: (1) thresholds based on the absolute value of an atmospheric window channel brightness temperature; (2) thresholds based on the difference between the brightness temperature in an atmospheric window channel and the brightness temperature centered on a strong water vapor absorption line; and (3) a threshold using the difference between the window channel brightness temperature and the tropopause temperature based on climatology. Simultaneous observations of these infrared identified DCCs with the Advanced Microwave Sounding UnitHumidity Sounder for Brazil (AMSU-HSB) using $183 \mathrm{GHz}$ water channels provide a statistical correlation with microwave deep convection and overshooting convection. In the past 10 years, the frequency of occurrence of DCCs has decreased for the tropical ocean, while it has increased for tropical land. The area of the tropical zone associated with DCCs is typically much less than $1 \%$. We find that the least frequent, more extreme DCCs show the largest trend in frequency of occurrence, increasing over land and decreasing over ocean.

The trends for land and ocean closely balance, such that the DCC frequency changed at an insignificant rate for the entire tropical zone. This pattern of essentially zero trend for the tropical zone, but opposite land/ocean trends, is consistent with measurements of global precipitation. The changes in frequency of occurrence of the DCCs are correlated with
\end{abstract}

the Niño34 index, which defines the sea surface temperature (SST) anomaly in the east-central Pacific. This is also consistent with patterns seen in global precipitation. This suggests that the observed changes in the frequency are part of a decadal variability characterized by shifts in the main tropical circulation patterns, which does not fully balance in the 10 -year AIRS data record. The regional correlations and anticorrelations of the DCC frequency anomaly with the Multivariate ENSO Index (MEI) provide a new perspective for the regional analysis of past events, since the SST anomaly in the Nino34 region is available in the form of the extended MEI from 1871 .

\section{Introduction}

Extremely cold deep convective clouds (DCCs) are frequently associated with severe weather and heavy precipitation events. A detection of a trend in the frequency of these clouds would have significant implication on our understanding of changes in the frequency of severe storms. However, while there are many papers on deep convective clouds, no studies of trends in frequency of deep convective clouds are found in the literature.

The association between severe weather and extremely cold clouds was first noted in $11 \mu \mathrm{m}$ thermal infrared images from Earth orbiting satellites three decades ago (Adler et al., 1979; Reynolds, 1980). The association between heavy rainfall and deep convective clouds has led to the development of a precipitation algorithm for GOES (Janowiak and Arkin, 1991; Joyce and Arkin, 1997). Cases where the brightness temperature at $6.7 \mu \mathrm{m}$ is warmer than at $11 \mu \mathrm{m}$ (e.g., Ackerman, 1996; Schmetz et al., 1997) were interpreted as 
convective overshooting clouds, providing further evidence of their association with deep convective storms. More recently, severe storms have been associated with the overshooting clouds, which are a subclass of DCCs (e.g., Setvak et al., 2008, 2012; Bedka et al., 2010). Damage associated with severe storm is well documented for relatively densely populated land areas. However, in the tropical zone, where severe storms are common, these events may be less striking and therefore less documented.

Satellite data have been used to identify DCCs and cloud tops that reach or overshoot the tropopause transition layer (TTL) (Fueglistaler et al., 2009; Gettelman et al., 2009), but these definitions are dependent on footprint size and the spectral channels available for the analysis. Thus Gettelman et al. (2002) selected DCCs based on a brightness temperature in the $11 \mu \mathrm{m}$ channel, $\mathrm{B} T_{11}<210 \mathrm{~K}$. These objects cover less than $1 \%$ of the tropical zone. They then identified those clouds that are colder than the temperature of the climatological tropopause cold point $(0.5 \%$ of the tropical zone) as "overshooting convection". Alcala and Dessler (2002) and Liu and Zipser $(2005,2008)$ identified $20 \mathrm{dBZ}$ precipitation features (PFs) with the $13.8 \mathrm{GHz}$ rain radar from TRMM (Tropical Rainfall Measuring Mission). Deep convection was associated with PFs above the level of neutral buoyancy (LNB), and overshooting convection was identified by PFs where the associated height exceeds the climatological tropopause height. Hong et al. (2005) used a set of inequalities derived from $183 \mathrm{GHz}$ sounder channels from AMSU-B (Advanced Microwave Sounding Unit) to identify $0.6 \%$ of the area of the tropical zone as deep convective clouds. Young et al. (2012) used $1 \mathrm{yr}$ (2007) of $95 \mathrm{GHz}$ CloudSat, CALIPSO (Cloud-Aerosol Lidar) and MODerate resolution Imaging Spectroradiometer (MODIS) data to show that $61 \%$ of the cold cloud features (CCFs) identified by the condition $\mathrm{B} T_{11}<210 \mathrm{~K}$ cover about $1 \%$ of the tropical zone and are associated with precipitation features from CloudSat at altitudes higher than $14 \mathrm{~km}$ (i.e., reach the bottom of the TTL).

The ability to detect these cold clouds in the infrared has been available for decades from the High Resolution Infrared Radiation Sounder (HIRS) and the Advanced Very High Resolution Radiometer (AVHRR). Wylie at al. (2005) published a study of trends in the frequency of high clouds using the IR data from a $25 \mathrm{yr}$ long series of HIRS. The high clouds covered about $40 \%$ of the area of the tropical zone, two orders of magnitude more than the frequency of DCCs. He found no significant trends. In view of the association of DCCs with heavy precipitation, the time series of tropical precipitation can be used as proxy for changes in the frequency of DCCs. The WCRP report on Global Precipitation (2008) finds no significant trend in precipitation between 1979 and 2004.

The objective of our study is to use the first $10 \mathrm{yr}$ of IR data from AIRS to evaluate trends in the frequency of DCCs in the tropical zone and to interpret the results using the correlation with the vertical pressure velocity at $500 \mathrm{hPa}$ and the
Nino34 index. In Sect. 2 we introduce the AIRS and AMSUHSB (Humidity Sounder for Brazil) data used in the analysis. In Sect. 3 we describe criteria for selecting DCCs. Section 4 presents trend results. In Sect. 5 we analyze correlations between the DCC frequency, vertical velocity and the Nino34 index from a climate perspective.

\section{Data}

AIRS is uniquely suited for the detection of a change in the DCC frequency. AIRS (together with MODIS) has been in orbit for the past $10 \mathrm{yr}$ with the expectation of a $20 \mathrm{yr}$ lifetime. The AIRS absolute calibration accuracy and stability have been well documented (Aumann et al., 2006, 2012). AIRS was launched in May 2002 on the EOS Aqua satellite into a $705 \mathrm{~km}$ altitude sun-synchronous, 98-degree inclination circular orbit. We refer to the 13:30 LT ascending node as the "day" overpass, and to the 01:30 LT descending part of each orbit as the "night" overpass. The ascending node and altitude of the orbit are actively maintained. The AIRS footprint subtends an angle of 1.1 degree. The footprints are scanned \pm 49.5 degrees cross-track resulting in a $1650 \mathrm{~km}$ wide swath. The footprint geometrical size on the ground increases from a spot of $13.5 \mathrm{~km}$ diameter at nadir to a $99 \mathrm{~km} \times 23 \mathrm{~km}$ ellipse at the extreme scan angles, with an average $30 \mathrm{~km}$ size averaged over all scan angles. On the same spacecraft as AIRS is the Humidity Sounder for Brazil (HSB) instrument (Lambrigtsen and Calheiros, 2003). Since the HSB has the same channels at $183 \mathrm{GHz}$ as AMSU-B on a number of NOAA spacecraft, we refer to HSB in the following simply as AMSU-HSB. It has the same 1.1-degree footprint size and scan pattern as AIRS. The AIRS and AMSUHSB scan patterns are synchronized, and their footprints are aligned to within 0.2 degrees (Lambrigtsen and Lee, 2003). AIRS and AMSU-HSB thus provide essentially simultaneous coverage of infrared data with $183 \mathrm{GHz}$ microwave data. The AMSU-HSB on EOS Aqua produced quality data only between 1 September 2002 and 4 February 2003.

Essentially un-interrupted AIRS data are available from 1 September 2002 to present. Each day, AIRS generates 3 million spectra, which are available from the GSFC/DISC in the form of 240 files, each of which corresponding to $6 \mathrm{~min}$ of data. In order to make these data more suitable to the analysis of climate related objectives, a subset of these data is collected in a single daily file, referred to as AIRS Calibration Data Subset (ACDS), also available from the GSFC/DISC. The ACDS contains AIRS, AMSU-A and AMSU-HSB calibration-related data, including all spectra between latitudes $50^{\circ} \mathrm{N}$ and $50^{\circ} \mathrm{S}$, where the brightness temperature in the atmospheric window channel at $1231 \mathrm{~cm}^{-1}$ (bt1231) is less than $225 \mathrm{~K}$. We refer to these data as cold cloud features $(\mathrm{CCFs})$, which are found on average in $2.3 \%$ of the tropical zone. Many CCFs are grouped in CCF clusters containing one or more DCCs. 


\section{Deep convective clouds selection and characterization}

From all CCFs, each day on average 22000 footprints associated with CCFs are found in the tropical zone, $30^{\circ} \mathrm{S}$ to $30^{\circ} \mathrm{N}$. In order to quantify the distance of the cloud tops from the tropopause, we use several AIRS channels and channel differences. The brightness temperatures (bt's) measured in these channels are denoted by their spectral response function center wave numbers in $\mathrm{cm}^{-1}$. Thus bt1231, bt900, and bt790 define the brightness temperatures at the shortwave start, the center, and longwave end of the $11 \mu \mathrm{m}$ atmospheric window channel, which extends from about $8.1 \mu \mathrm{m}$ $\left(1231 \mathrm{~cm}^{-1}\right)$ to $12.7 \mu \mathrm{m}\left(790 \mathrm{~cm}^{-1}\right)$. The bt1419 refers to the water vapor sounding channel at $1419 \mathrm{~cm}^{-1}$. Under cloudfree tropical conditions, this channel sounds near $12 \mathrm{~km}$ altitude. We will also use the difference DTW = bt1231-bt1419, which has typical values in the range of $65 \mathrm{~K}$ to $75 \mathrm{~K}$ under clear-sky tropical conditions. In the following we first discuss the method that will be used to select cold clouds based on the proximity of their cloud tops to the tropopause. We then discuss the relationship of the selected objects to deep convection or overshooting convection based on simultaneous observations with AMSU-HSB and on results from the literature.

\subsection{DCC selection}

Conceptually, there are three methods to identify cold clouds near the tropopause: (1) using a fixed brightness temperature threshold, (2) using a threshold relative to the climatological tropopause temperature and (3) using a threshold based on the difference between a surface channel and a sounding channel. The first two methods are discussed mostly for their legacy value and to allow a comparison of results with the third method, which we use in this study.

1. The legacy method in the infrared for the selection of DCCs uses a fixed brightness temperature threshold for the broad $11 \mu \mathrm{m}$ atmospheric window channel, $T_{\mathrm{B} 11}$, typically $210 \mathrm{~K}$ (Mapes and House, 1993; Gettelman et al., 2002). As noted above the $11 \mu \mathrm{m}$ window actually extends from about $8.1 \mu \mathrm{m}\left(1231 \mathrm{~cm}^{-1}\right)$ to $12.7 \mu \mathrm{m}\left(790 \mathrm{~cm}^{-1}\right)$. The properties of the DCCs identified by fixed $T_{\mathrm{B} 11}$ threshold vary with the latitude, longitude, and the composition of the cloud top. Figure 1 shows the latitude dependence of the tropical tropopause cold point temperature, $T_{\text {Trop }}$, as a mean over 1981-2010 data from the National Centers of Environmental Prediction (NCEP) Reanalysis (Kalnay et al., 1996, available from NOAA/OAR/ESRL PSD, Boulder, Colorado, USA, http://www.esrl.noaa.gov/ $\mathrm{psd} /$ ). $T_{\text {Trop }}$ varies zonally and seasonally from $193 \mathrm{~K}$ to $212 \mathrm{~K}$. In addition, $T_{\mathrm{B} 11}$ corresponds to the temperature at which the cloud becomes optically thick at $11 \mu \mathrm{m}$. From Fig. 1 it is clear that there is no simple relationship between cloud top temperature and the

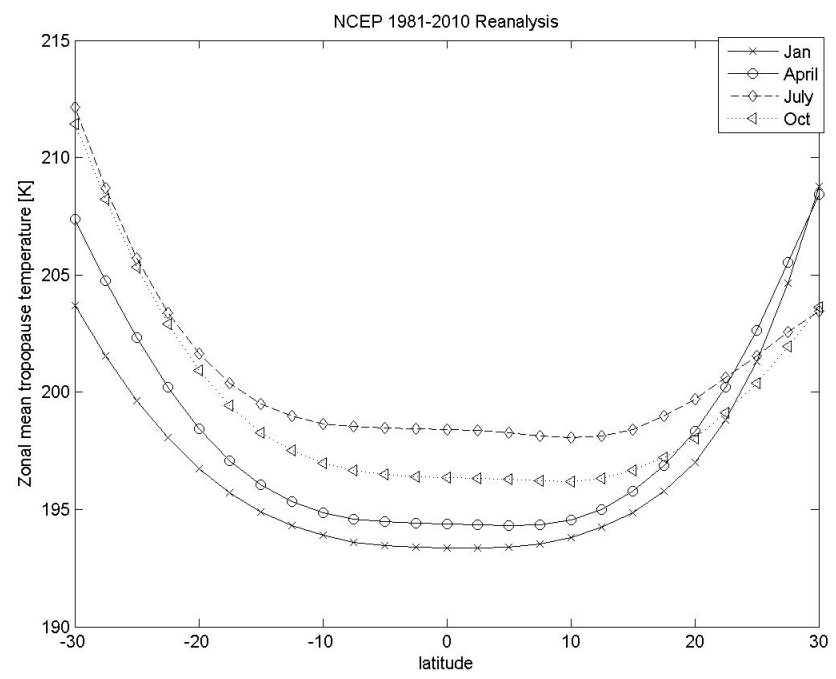

Fig. 1. Latitude dependence of the tropical tropopause cold-point temperature based on the NCEP 1981-2010 Reanalysis.

climatological tropopause cold point temperature. Aumann et al. (2011) modeled the tops of cold clouds as uniform slabs of ice clouds using a range of particle sizes and densities at various heights relative to the tropopause, where the cloud properties were fitted to match the observed slope across the $11 \mu \mathrm{m}$ window. At the center of the $11 \mu \mathrm{m}$ window, these clouds become optically thick at about $20 \mathrm{hPa}$ below the physical cloud top. For a tropical profile at $100 \mathrm{hPa}$, this corresponds to a penetration depth into the physical cloud top of about $1 \mathrm{~km}$, corresponding to about $4 \mathrm{~K}$. This means that the DCCs selected with $T_{\mathrm{B} 11}<210 \mathrm{~K}$ have clouds tops at $206 \mathrm{~K}$ : they are well below the tropopause, except near $30^{\circ} \mathrm{N}$ and $30^{\circ} \mathrm{S}$.

2. The third method for the identification of DCCs is based on a threshold relative to the climatological tropopause temperature. Rather than using dynamic time warping (DTW) for the selection of DCCs and making an indirect case for the proximity of the cloud tops to the tropopause, DCCs can be identified by the difference between $T_{\mathrm{B} 11}$ and $T_{\text {Trop. }}$. Gettelman et al. (2002) used the condition $T_{\mathrm{B} 11}-T_{\text {Trop }}<0$ to identify clouds overshooting the tropopause. Here we use the bt $900-T_{\text {Trop }}<2$ threshold in order to account for the penetration depth of the $900 \mathrm{~cm}^{-1}$ signal into the cloud top. The drawback of using $T_{\text {Trop }}$ directly is that it fails to account for local variations of the actual tropopause cold point temperature from the climatological $T_{\text {trop }}$, which correlate with deep convection.

3. The third method, using a threshold based on the difference between a surface channel and a sounding channel, was first used with $T_{\mathrm{B} 11}$ and the broad $6.7 \mu \mathrm{m}$ water channel, $T_{\mathrm{B} 6.7}$ (e.g., Ackerman, 1996, Schmetz 
Table 1. Percent of DCCs identified with various IR brightness temperature thresholds and differences, segregated by surface type and time of day, from AIRS data in 2002-2012.

\begin{tabular}{llccccc}
\hline & Threshold & Tropical zone & Ocean day & Ocean night & Land day & Land night \\
\hline DCC210 & bt900<210K & 0.800 & 0.557 & 0.650 & 0.932 & 1.09 \\
DCCw0 & bt1231-bt1419<0 & 0.644 & 0.501 & 0.629 & 0.847 & 1.01 \\
DCCw4 & bt1231-bt1419 $\leq 4$ & 0.158 & 0.107 & 0.184 & 0.235 & 0.165 \\
DCC200 & bt900<200K & 0.140 & 0.092 & 0.161 & 0.197 & 0.139 \\
DCCt2 & bt900- $T_{\text {Trop }}<2$ & 0.061 & 0.031 & 0.070 & 0.072 & 0.130 \\
\hline
\end{tabular}

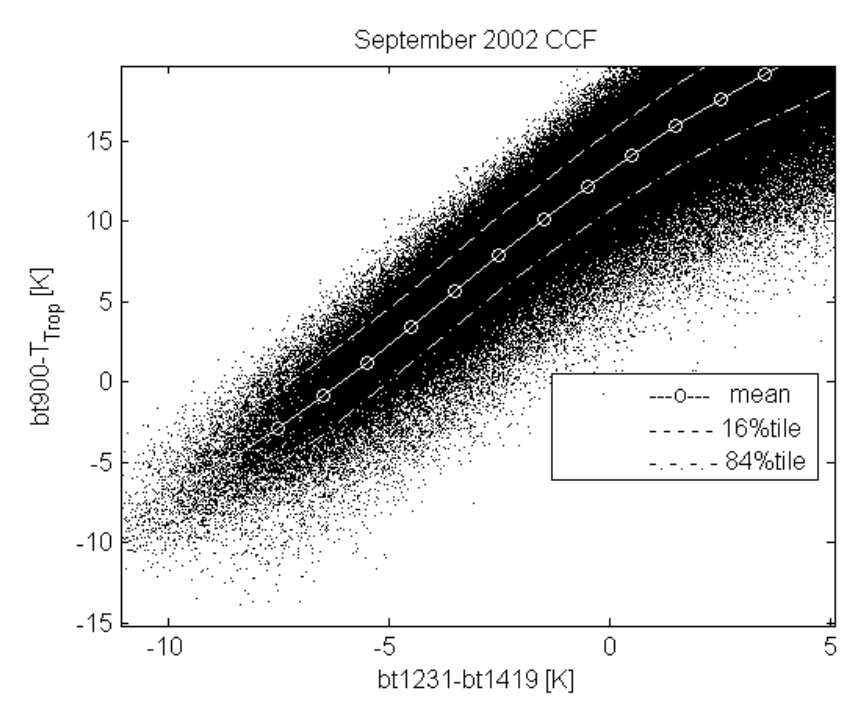

Fig. 2. The relationship between the $\mathrm{DTR}=\mathrm{bt} 900-T_{\text {Trop }}$ and DTW $=$ bt1231-bt1419 is illustrated in a scatter diagram for all CCFs collected in September 2002 with DTR < 20 K.

et al., 1997). The brightness temperature $T_{\mathrm{B} 6.7}$ refers to a layer where the atmosphere becomes opaque due to the combined opacity of water vapor or clouds. In the presence of optically thick clouds and without water vapor, $T_{\mathrm{B} 11}$ and $T_{\mathrm{B} 6.7}$ would be nearly identical. With water vapor, the absorption due to water vapor in the region located between the pressure level at which the cloud becomes optically thick and the tropopause is counteracted by the emission from water vapor above the tropopause in the warmer stratosphere. Historically, clouds with $T_{\mathrm{B} 11}-T_{\mathrm{B} 6.7}<0$ were assumed to overshoot the tropopause (Ackerman 1996). With AIRS data we use the difference between the extremely clear window channel bt1231 and the very strong water vapor channel bt1419, which is conceptually similar to $T_{\mathrm{B} 11}-T_{\mathrm{B} 6.7}$, but avoids the ambiguity due to wide bandwidth. Figure 2 illustrates the relationship between the differences defined as DTR $=$ bt900$T_{\text {Trop }}$ and DTW $=$ bt1231 - bt1419 in a scatter plot for all CCFs collected in September 2002. Calculated separately and shown in white are the mean, and the 16 and 84 percentiles of the distribution function for $2 \mathrm{~K}$ wide DTW bins. About $0.6 \%$ of the area of the tropical zone satisfies the condition DTW $<0$, but DTR is typically $+12 \mathrm{~K}$ (i.e., the cloud tops are well below the tropopause). Still, about $30 \%$ of the objects selected using the condition DTW $<0$ also satisfy the condition DTR $<4$ meaning that the physical cloud tops are above the tropopause. We use the condition DTR $<4 \mathrm{~K}$ to make an approximate adjustment for the $4 \mathrm{~K}$ penetration depth. Typically $0.16 \%$ of the area of the tropical zone is covered with DCCs identified by the condition DTW $\leq 4 \mathrm{~K}$, and $80 \%$ of these clouds $(0.12 \%$ of the area) have bt1231 lower than the brightness temperature of the climatological tropopause. The mean of the distribution function shown in Fig. 2 reveals an empirical relationship between DTW and DTR, which we use to select DCCs based on their proximity to the local tropopause.

Table 1 summarizes the results of application of three methods of DCC selection described above. The first column in Table 1 denotes the type of the DCCs, and the second column defines the threshold. For example, DCC210 is the set of all cold clouds from the tropical zone that satisfy the condition bt900 < $210 \mathrm{~K}$. Cold clouds selected with the DTW $<0$ and DTW $\leq 4 \mathrm{~K}$ thresholds are referred to as DCCw0 and DCCw4, respectively. The same threshold is applied to all footprints between -49 and +49 degrees from nadir. We defined DTR $=$ bt900- $T_{\text {Trop }}$ to emulate the legacy definition $T_{\mathrm{B} 11}-T_{\text {Trop }}$, but the statistical analysis for the DCCt 2 uses DTR $<2$ threshold to account for the cloud optical depth. The ratio of the count of footprints selected by the thresholds, divided by the count of all footprints in a given area, multiplied by 100 , represents the percent of the area covered by these objects. We identify this ratio with the DCC frequency of occurrence, or simply the DCC frequency. The 3 rd through 7 th columns present the $10 \mathrm{yr}$ mean DCC frequencies for the entire tropical zone subdivided into day/night and land/ocean. In Table 2 we included the legacy DCC210, DCC200 and DCCt 2 only for comparison with the DCCw0 and DCCw4, which are the focus of our study. 
Table 2. Percent of DCCs identified in the IR with AMSU-HSB deep convection and overshooting convection for September 2002, November 2002 and January 2003.

\begin{tabular}{lcccccc}
\hline & \multicolumn{3}{c}{$\begin{array}{c}\text { Percent AMSU-HSB } \\
\text { deep convection }\end{array}$} & \multicolumn{3}{c}{ Percent AMSU-HSB } \\
& Sep & Nov & Jan & Sep & Nov & Jan \\
\hline DCC210 & 48 & 47 & 46 & 20 & 18 & 17 \\
DCCw0 & 53 & 50 & 50 & 22 & 20 & 20 \\
\hline DCCw4 & 72 & 68 & 69 & 39 & 35 & 36 \\
DCC200 & 67 & 66 & 64 & 38 & 32 & 31 \\
\hline DCCt2 & 71 & 72 & 77 & 45 & 43 & 49 \\
\hline
\end{tabular}

\subsection{DCC selection verification}

From the IR data alone, we only know the frequency of DCCs in the tropical zone as function of the proximity of cloud tops (defined by the unit optical depth at $1231 \mathrm{~cm}^{-1}$ ) to the tropopause. DCCw0 clouds cover $0.6 \%$ of the tropical zone, and about $30 \%$ of them have cloud tops above $T_{\text {Trop }}$. DCCw4 clouds cover $0.16 \%$ of the tropical zone, and $80 \%(0.12 \%$ of the area of the tropical zone) have cloud tops overshooting the climatological tropopause. In order to interpret the DCC frequency as the frequency of severe storms, we have to verify that the cold clouds selected with AIRS using the purely radiometric inequality bt $1231-$ bt $1419 \leq 4 \mathrm{~K}$ are not simply optically thick ice clouds, but are with high probability associated with deep or overshooting convection. For this we use observation with other instruments directly or indirectly.

For the direct verification, we use the set of inequalities derived by Hong et al. (2005) for the three $183 \mathrm{GHz}$ water channels from AMSU-B on NOAA polar orbiters. During September 2002, while AMSU-HSB on EOS Aqua was in operation, $53 \%$ of all DCCw0 identified using the infrared alone were identified as microwave deep convective, and $22 \%$ as microwave overshooting convection. This fraction increased to $72 \%$ for the DCCw4, with $39 \%$ identified as microwave deep and overshooting convection. Table 2 summarizes this information for the cited and other detection thresholds and also includes results from November 2002 and January 2003 data. For each month about 200000 DCC210 were identified.

A comparison of the detection frequency of deep convection using other observing systems provides an indirect verification. Hong et al. (2005) used NOAA 15, 16, and 17 AMSU-B $183 \mathrm{GHz}$ channels trained on TRMM data to show that $0.3 \%$ of the area of the tropics is associated with deep convection, and $0.08 \%$ of the area is overshooting convection. These frequencies are almost exactly the frequencies expected from our direct verification of DCCw0 using AMSUHSB on EOS Aqua. Liu and Zipser (2007) used $13.8 \mathrm{GHz}$ Precipitation Radar (PR) and Visible and Infrared Scanner (VIRS) data from TRMM. They found that in the $20^{\circ} \mathrm{N}$ to
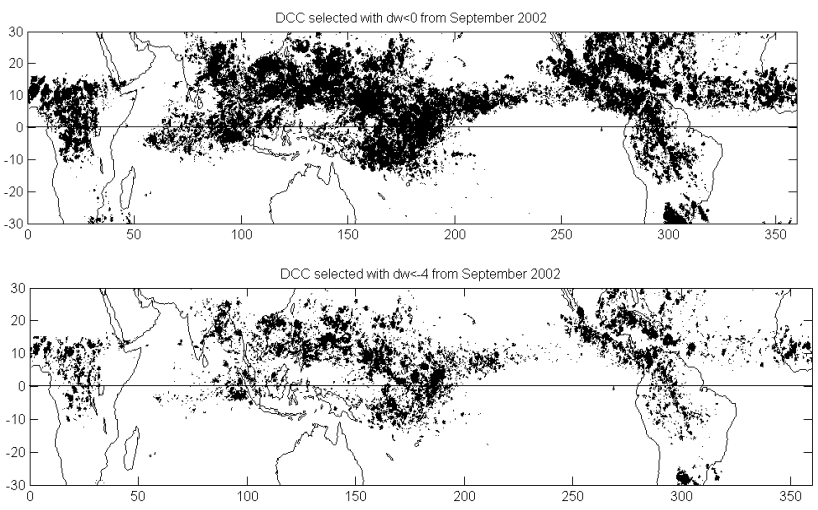

Fig. 3. Top: CCFs that satisfy the condition DTW $<0$. Bottom: CCFs that satisfy the condition DTW $\leq 4$.

$20^{\circ} \mathrm{S}$ zone where $57 \%$ of the clouds satisfy $T_{\mathrm{B} 11}<210 \mathrm{~K}$, rain was detected by the PR near the surface. This is roughly consistent with our finding that $50 \%$ of the DCC210 are associated with AMSU-HSB deep convection based on data from the $30^{\circ} \mathrm{N}$ to $30^{\circ} \mathrm{S}$ zone. Young et al. (2012) made use of the $95 \mathrm{GHz}$ cloud radar from CloudSat, the lidar from CALIPSO and MODIS data to gain insight into the meaning of the $T_{\mathrm{B} 11}-T_{\mathrm{B} 6.7}<0$ threshold. They concluded that $61 \%(55 \%)$ of the objects selected with the $T_{\mathrm{B} 11}<210 \mathrm{~K}$ $\left(T_{\mathrm{B} 11}-T B_{6.7}<0\right)$ threshold are associated with deep convection that reaches $14 \mathrm{~km}$ altitude (i.e., the base of the tropopause transition layer (TTL)). This is consistent with the frequency found using our DCC210 and DCCw0 thresholds.

Figure 3 shows the global distribution of the $\mathrm{DCCw} 0$ and DCCw4 for September 2002. There is a high degree of spatial correlation between DCCw0 and DCCw4. This suggests that DCCw4 are tropopause-penetrating clouds, which are surrounded by $\mathrm{DCCw} 0$ at the center of even larger clusters of CCFs.

In the Sect. 4 we analyze trends in the frequency of DCCw0 and DCCw4 in 2002-2012. For consistency with legacy definitions, we also evaluate trends for DCC210 and DCC200, which satisfy the simple bt $900<210 \mathrm{~K}$ and $<200 \mathrm{~K}$ conditions, respectively. The bt $900-T_{\text {Trop }}<2 \mathrm{~K}$ threshold relative to the climatology tropopause temperature defines the least frequent class of DCCs, DCCt2.

\section{Trend analysis}

In the following we illustrate the 10-year trend analysis using the DCCw0. The top panel in Fig. 4 shows the daily DCCw0 fraction for the tropical zone. Each dot represents the day/night averaged area fraction. The same data are shown in the bottom panels, smoothed with a 96-day running mean. The smoothing is used only to illustrate the seasonal pattern better, and the data processing uses the daily values. 
Table 3. Summary of trends and trend uncertainties in $\% \mathrm{yr}^{-1}$ units.

\begin{tabular}{lllcccc}
\hline & Threshold & Tropical zone & Ocean day & Ocean night & Land day & Land night \\
\hline DCC210 & bt $900<210 \mathrm{~K}$ & $+0.042 \pm 0.12$ & $-0.72 \pm 0.17$ & $-1.20 \pm 0.17$ & $+1.82 \pm 0.22$ & $+1.86 \pm 0.24$ \\
DCCw0 & bt1231-bt1419<0 & $+0.038 \pm 0.10$ & $-0.46 \pm 0.16$ & $-1.01 \pm 0.16$ & $+1.73 \pm 0.21$ & $+1.78 \pm 0.21$ \\
DCCw4 & bt1231-bt1419 $\leq 4$ & $+0.15 \pm 0.17$ & $-0.82 \pm 0.27$ & $-1.30 \pm 0.25$ & $+2.75 \pm 0.33$ & $+2.03 \pm 0.32$ \\
DCC200 & bt900<200K & $-0.46 \pm 0.21$ & $-1.83 \pm 0.32$ & $-2.04 \pm 0.30$ & $+2.55 \pm 0.36$ & $+1.89 \pm 0.36$ \\
DCCt2 & bt900- $T_{\text {Trop }}<2$ & $-0.58 \pm 0.29$ & $-2.41 \pm 0.47$ & $-2.13 \pm 0.40$ & $+2.35 \pm 0.53$ & $+2.51 \pm 0.49$ \\
\hline
\end{tabular}

Table 4. Trends for tropical oceans.

\begin{tabular}{lcccc}
\hline & West Pacific & East Pacific & Indian Ocean & Atlantic Ocean \\
& $120^{\circ} \mathrm{E}-200^{\circ} \mathrm{E}$ & $200^{\circ} \mathrm{E}-280^{\circ} \mathrm{E}$ & $30^{\circ} \mathrm{E}-120^{\circ} \mathrm{E}$ & $280^{\circ} \mathrm{E}-30^{\circ} \mathrm{E}$ \\
\hline DCCw0 trend [\% $\mathrm{yr}^{-1}$ ] & $-1.79 \pm 0.27$ & $-1.51 \pm 0.35$ & $+1.05 \pm 0.29$ & $+1.71 \pm 0.39$ \\
Mean DCCw0 frequency [\%] & 1.04 & 0.29 & 0.78 & 0.24 \\
Relative area & $28 \%$ & $27 \%$ & $25 \%$ & $20 \%$ \\
\hline
\end{tabular}
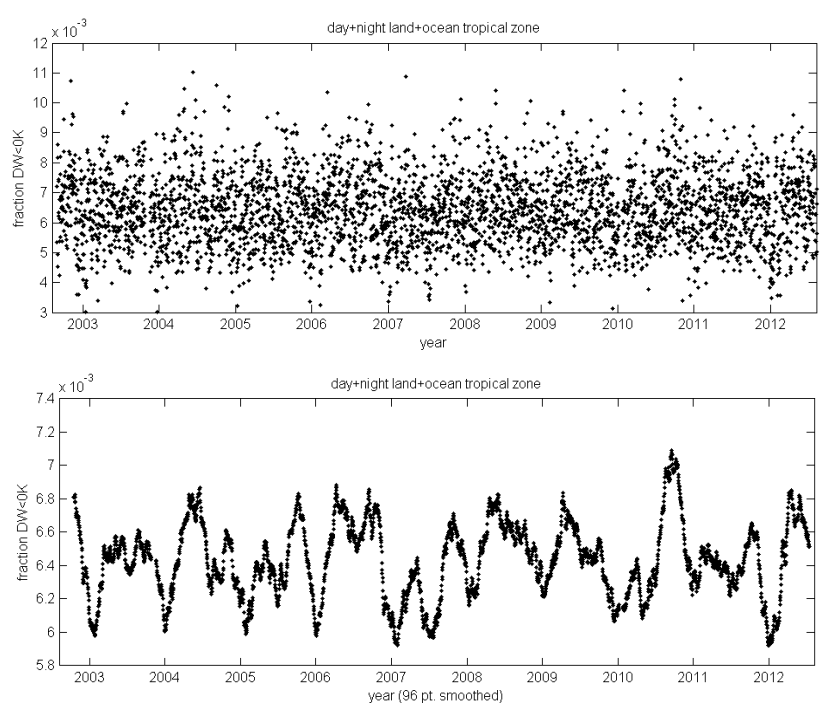

Fig. 4. The daily DCCw0 fraction for the tropical zone. Top: daily points. Bottom: the same points smoothed with 96-day running mean.

We use 96-day smoothing since it is a multiple of the 16-day EOS Aqua orbit repeat cycle. All data shown in the following are presented after smoothing. The small annual variability is clearly seen in the relatively quiet period between 2003 and 2007. Thereafter, it is overshadowed by El Niño-Southern Oscillation (ENSO) episodes. When the land and ocean area fractions are overlaid (shown in Fig. 5 for the night data), it becomes apparent that (normalized to area) there are more DCCs over land than over ocean. This observation is consistent with rain radar measurements of precipitation in the tropics (Liu and Zipser 2005). The frequency of DCCs over land has been increasing, while it has been decreasing over ocean. Part of the variability seen in Fig. 5 is the seasonal variability,

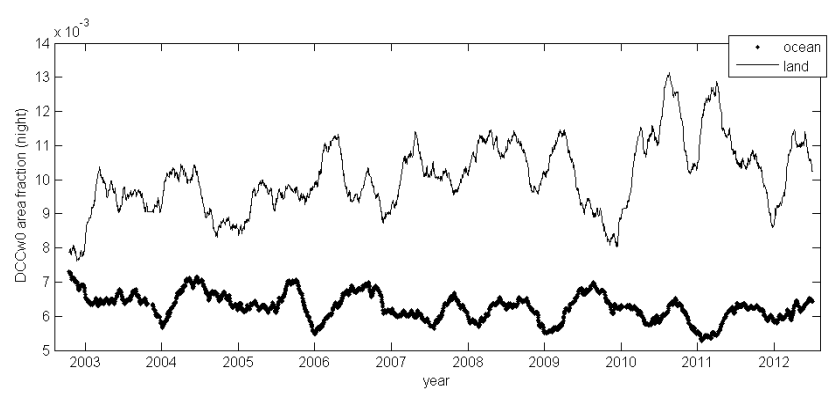

Fig. 5. The night land (solid) and ocean (bold trace) DCCw0 area fractions as function of time.

which is less than $10 \%$ of the mean, with clear minima for the NH winters, and not so clear maxima in the NH summer. Superimposed on the seasonal variability is a bigger ENSO signal. The anomaly is defined as the difference between the daily data and the least-squares fit of the data to a loworder harmonic series. This removes the seasonal variability. Numerical details of this procedure and the estimation of the anomaly trend and trend uncertainty are given in Appendix A1. The plot of the frequency of DCCw4 as function of time looks very similar to the frequency of DCCw0 shown in Fig. 5, but with reduced frequencies and consequently larger noise. For this reason we use the DCCw0 anomaly for the correlation with other anomalies. Figure 6, which shows the anomalies for DCCw0 identified over land and ocean (heavy trace), confirms the land/ocean frequency anticorrelation $(r=-0.70)$, which is visually obvious in Fig. 5. The anomaly trend for ocean is $-0.46 \pm 0.16 \% \mathrm{yr}^{-1}$; for land it is $+1.78 \pm 0.21 \% \mathrm{yr}^{-1}$, where the stated uncertainties correspond to $1 \sigma$ confidence level. Table 3 lists the anomaly trends and the trend uncertainties for the tropical day/night average, land/ocean, day/night, for the various DCC thresholds matching Table 1 . Tables 4 and 5 show the DCCw0 


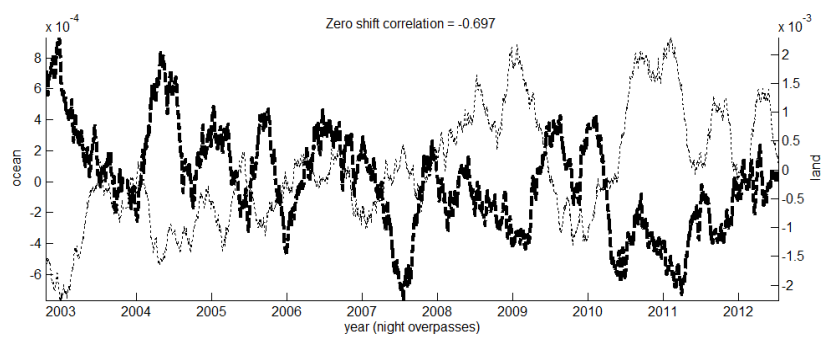

Fig. 6. DCCw0 fraction anomalies of the night overpass data shown in Fig. 5. Bottom: DCCw0 fraction anomalies of the day overpass data. The bold trace refers to ocean; the light trace is for land.

mean frequencies and frequency trends subdivided into four tropical oceans (east Pacific, west Pacific, Indian and Atlantic oceans) and three land areas (Americas, Africa, and India/Australia). The split of the Pacific into the west and east basin has been done to test whether the DCC trends are different over the so-called ENSO centers of action.

\section{Discussion}

Before discussing the observed trends, we would like to remind the reader which variables were subjected to the trend analysis: we select cold clouds at or above the tropopause using the AIRS IR data (Sect. 3). We identify DCCs using thresholds relative to bt900, the difference of bt1231-bt1419, and the difference between bt900 and climatological tropopause temperature. Of the DCCs identified using the infrared alone, $53 \%$ of the DCCw0 and $72 \%$ of the DCCw4 are also identified by simultaneous AMSUHSB measurements as microwave deep convection, and $22 \%$ of the DCCw0 and $39 \%$ of the DCCw4 match the criteria for microwave deep convection and overshooting convection. The trends deduced from the DCCs selected using AIRS IR data statistically pertain to deep convection. However, $47 \%$ of the DCCw0 and $28 \%$ of the DCCw4 selected using AIRS IR data alone are not associated with microwave deep or overshooting convection. This explains observations by Bedka et al. (2010) and Setvak et al. (2012), who noted that in detailed case studies of cold clouds selected using the MODIS channels M31 and M27, the $T_{\mathrm{m} 31}-\mathrm{TB}_{\mathrm{m} 27}<0$ threshold identifies not only overshooting cloud tops but almost the entire convective anvil cloud.

The changes in the DCC frequency with time, summarized in Table 3, show several interesting features.

\subsection{Trends over land and ocean}

We find an almost total absence of a trend on a global scale, but strongly anti-correlated trends for land and ocean. Note that while the $10 \mathrm{yr}$ mean frequency of the DCCw0 is four times larger than the frequency of DCCw4 (Table 2), neither set has a significant trend for the entire tropical zone.
However, the DCCw0 and DCCw4 trend normalized to frequency for day/night ocean/land are highly significant and differ by about a factor of 2, with the DCCw4 having consistently faster trends than DCCw0. The DCC frequency trends nearly cancel for the global average, except for the DCCt2 (tropopause overshooting) group, where the decreasing global trend is marginally significant. DCCs correlate with intense precipitation events. The observed tropical zone, land and ocean trends in the DCC frequency are consistent with the WCRP report on trends in global precipitation (2008). That report finds no significant trend in precipitation between 1979 and 2004 and also notes the anticorrelation between land and ocean. The $10 \mathrm{yr}$ time span of the AIRS data is too short to observe the reversal of the observed land/ocean trend for DCCs, which is expected based on the precipitation record.

The large magnitude and opposite signs of regional trends rule out instrumental artifacts. But when the trend gets closer to zero, even small systematic instrument trends have to be taken into consideration. Since DCCs are threshold identified, an instrument trend could introduce an artificial trend in the count of DCCs. The trend in bt1231 and bt900, which is measured relative to ocean buoys under clear tropical ocean conditions, has been less than $0.01 \mathrm{~K} \mathrm{yr}^{-1}$ (Aumann et al., 2012). If, due to an instrument trend, the bt $900<210 \mathrm{~K}$ threshold were to change over $10 \mathrm{yr}$ to become effectively a bt $900<211 \mathrm{~K}$ threshold, the frequency of DCCs would increase from $0.8 \%$ to $0.89 \%$ (i.e., a $10 \%$ increase per degree $\mathrm{K}$ ). This corresponds to a trend artifact of less than $10 \cdot 0.01 \% \mathrm{yr}^{-1}=0.1 \% \mathrm{yr}^{-1}$. Since this effect is smaller than the observed trend uncertainties, it can safely be neglected.

\subsection{DCC correlation with ENSO}

The frequency anomaly patterns, shown in Fig. 5 for the nighttime data over the Pacific Ocean, and the patterns for DCCs determined by other thresholds (not shown), suggest a correlation with the El Niño-Southern Oscillation (ENSO). To quantify the correlation we employ the Multivariate ENSO Index (MEI, Wolter and Timlin, 1998), which is often used for the analysis of inter-annual variability and trends in climate studies. The MEI is available as a monthly time series from http://www.esrl.noaa.gov/psd/enso/mei/table.html. It is closely related to the surface temperature anomaly of the Niño34 region in the central Pacific Ocean, normalized to zero mean and unity standard deviation for the values between 1950 and 1993. The MEI for the past $10 \mathrm{yr}$ has had a small but significant negative trend of $-0.13 \pm 0.04 \mathrm{~K} \mathrm{yr}^{-1}$. Figures 7 shows an overlay of the nighttime ocean and land $\mathrm{DCCw} 0$ frequency anomalies and the MEI (heavy trace). The correlation coefficient between DCC and MEI time series is +0.64 . For land, the MEI and the DCC frequency are strongly anti-correlated, $r=-0.67$. The observed correlations and anti-correlations are also seen in quasi-bi-annual 
Table 5. Trends for tropical lands.

\begin{tabular}{lccc}
\hline & Americas & Africa & India/Australia \\
\hline DCCw0 trend $\left[\% \mathrm{yr}^{-1}\right.$ ] & $+0.76 \pm 0.029$ & $+1.18 \pm 0.022$ & $+3.15 \pm 0.030$ \\
Mean DCCw0 frequency [\%] & 1.16 & 0.849 & 0.862 \\
Relative area & $28 \%$ & $34 \%$ & $37 \%$ \\
\hline
\end{tabular}
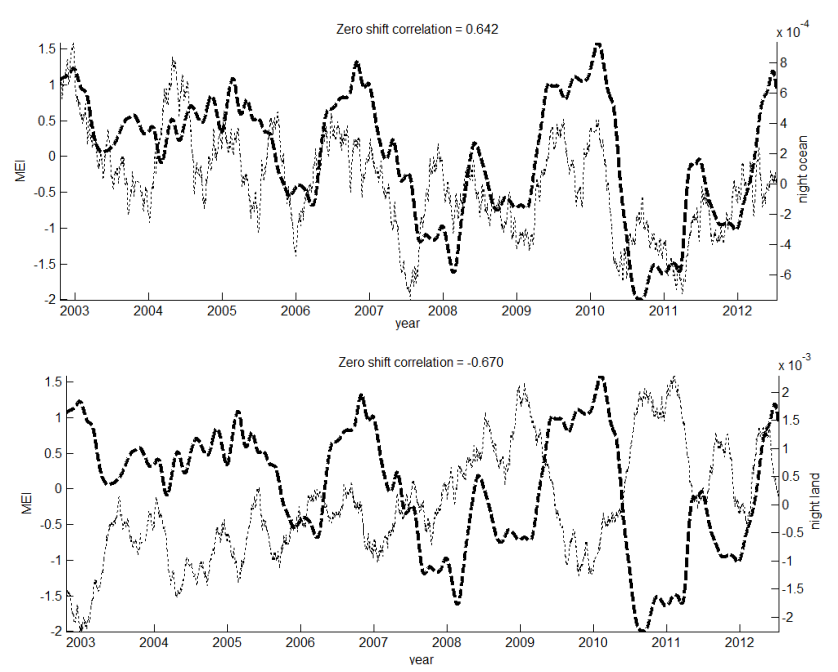

Fig. 7. The DCCw0 anomaly (light trace) and the MEI (heavy trace). Top panel presents ocean night. Bottom panel presents land night.

patterns. The correlation between the day ocean DCCw0 anomaly and the MEI is small $(r=+0.25)$. This can be explained by the regional analysis. The regional patterns in DCCw0 frequency trends, listed in Tables 3 and 4, are consistent with the classical picture of circulation cells in the tropical zone (Webster, 1983). For the Atlantic Ocean and Indian Ocean, the DCCw0 anomaly and MEI are anti-correlated ( $r=-0.60$ and $r=-0.63$, respectively), while the correlation is positive ( $r=+0.80$ ) for the Pacific Ocean (Fig. 8). The DCC anomaly trends over Indian and Atlantic oceans have the same sign as tropical land areas (Africa and Americas), because the main circulation cells related to these oceans originate from Africa and the Americas. The correlation between the MEI and the DCC anomalies points to an interesting perspective of the regional analysis of storms and heavy precipitation in the past, since the sea surface temperature (SST) anomaly in the Niño34 region is available in the form of the extended MEI from 1871 (Wolter and Timlin, 2011).

\subsection{DCC correlation with ascending air motion}

The relationship between ascending air, clouds and convection is numerically represented in models by the vertical velocity. Bony et al. (2004) suggested using the $500 \mathrm{hPa}$ vertical velocity, $\omega_{500}$, as a proxy for the large-scale vertical motions

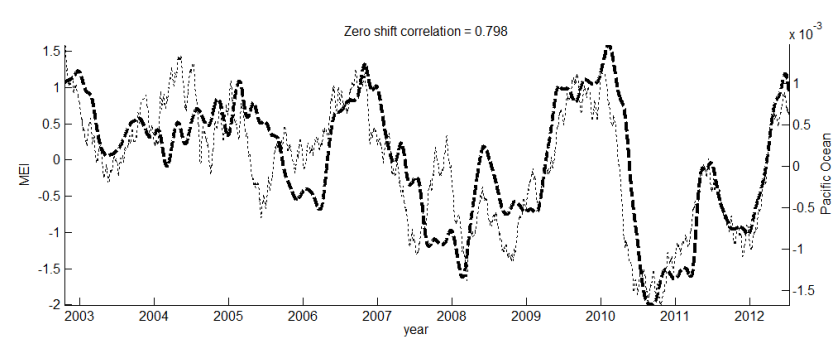

Fig. 8. The DCCw0 anomaly for averaged day/night over Pacific Ocean and the MEI (heavy trace).

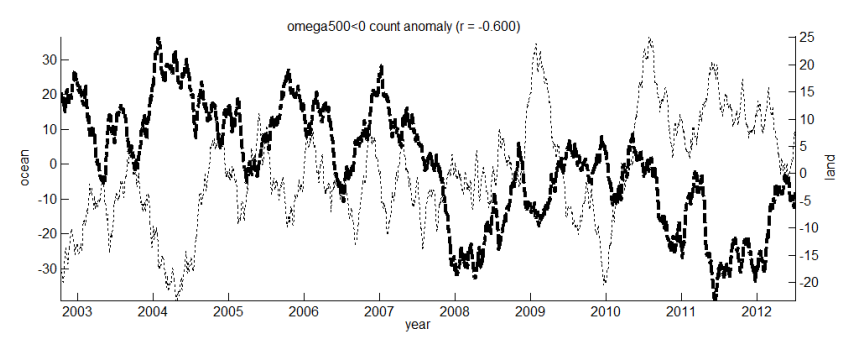

Fig. 9. Anomalies of the daily (day + night overpasses) count of the 2.5 degree cells identified with $\omega_{500}<0$ for ocean (bold) and land.

in the atmosphere. The vertical velocity is calculated daily on a global $2.5 \times 2.5$ degree $^{2}$ grid by the National Centers for Environmental Prediction (NCEP) Reanalysis. We refer to its value at $500 \mathrm{hPa}$ as $\omega_{500}$. By convention the negative $\omega_{500}$ corresponds to ascending motions. In order to test the role of $\omega_{500}$ in relation to the DCC frequency anomaly, we divide the data into land and ocean areas with negative $\omega_{500}$. Although an ascending air motion does not associate only with DCCs, a decrease in the area of negative $\omega_{500}$ can generally be interpreted as a decrease in convective cloudiness. For the past 10 years, on average $75 \%$ of the tropical ocean and $15 \%$ of tropical land area were associated with $\omega_{500}<0$. Figure 9 shows the anomaly of the daily count (day + night overpasses) of 2.5-degree cells identified with $\omega_{500}<0$. The time series over land and ocean are anti-correlated $(r=-0.60)$. The fraction of the ocean with $\omega_{500}<0$ has decreased for ocean at the rate of $-0.33 \pm 0.024 \% \mathrm{yr}^{-1}$, indicating a decrease in convection, while for land it has increased at the rate of $+0.5+/-0.05 \% \mathrm{yr}^{-1}$, indicating an increase in convection. Figure 10 shows the overlay of the land (top panel) and ocean (bottom panel) of the DCCw0 anomaly, both day + night averaged, on the $\omega_{500}<0$ anomaly. The trends in 

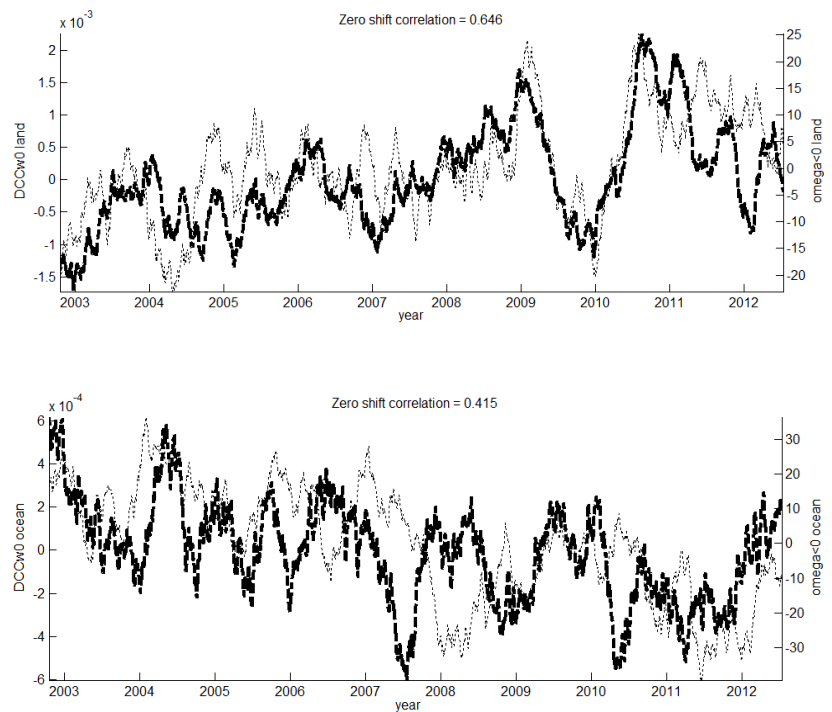

Fig. 10. The land (top panel) and ocean (bottom panel) of the DCCw0 anomaly compared with the $\omega_{500}<0$ anomaly.

the DCCw0 frequency and the area associated with $\omega_{500}<0$ are correlated: not surprisingly the trends in convection and deep convection are correlated. However, the DCCw0 frequency over land increased at the rate of $+1.5 \pm 0.2 \% \mathrm{yr}^{-1}$, three times faster than the area of $\omega_{500}<0$, which increased at the rate of $+0.5+/-0.05 \% \mathrm{yr}^{-1}$. For ocean the DCCw0 decreased at the rate of $-0.73 \pm 0.14 \% \mathrm{yr}^{-1}$, which is twice as fast as the area of $\omega_{500}<0$ decrease $\left(-0.33 \pm 0.024 \% \mathrm{yr}^{-1}\right)$.

\subsection{Trend balance between tropical land and ocean}

The highly significant DCC trends for the tropical oceans are almost exactly counterbalanced (area weighted) by the equally significant but opposite in sign tropical land trends. Table 3 shows that this balance is independent of the criteria for DCC identification. The anomaly trend for the entire tropical zone, expressed as percentage of the mean, ranges from $+0.1 \pm 0.07 \% \mathrm{yr}^{-1}$ to $+0.038 \pm 0.10 \% \mathrm{yr}^{-1}$ depending on which DCC group is selected. Generally speaking, the near-cancelation of the large positive land $(25 \%$ of the area of the tropical zone) trend by the negative ocean ( $75 \%$ of the tropical zone) trend cannot be caused by mass conservation: an increasing (decreasing) ascent must be balanced with increasing (decreasing) descent. However, due to the complexity and variability of tropical circulation, this general law of balance does not tell us where the anomalous ascent or descent occurs. Our study specifically shows the evolution of the anomalies in different regions, which is important for understanding the variability of tropical circulation.

The observation that the increase in the land DCC frequency is counterbalanced by the decrease in the ocean DCC frequency, combined with the regional correlation of the DCC frequency anomaly with the MEI, the correlation of the
DCC frequency anomaly with the $\omega_{500}<0$ anomaly, and the $\omega_{500}<0$ land/ocean trend anti-correlation, suggests a simple interpretation: the observed changes may be part of a decadal variability that shifts the distribution of DCCs in the tropics. An association of the changes only with ENSO may be an oversimplification, since the trends of the centers of ENSO action, approximated by the division of the Pacific Ocean into east and west, are almost the same. The increase in the DCC frequency for land has been matched for the past $10 \mathrm{yr}$ by a decrease for ocean.

Since DCCs are associated with intense precipitation, the increase in the frequency of DCCs for land results in an increase in precipitation over land, which is counter-balanced by a decrease in precipitation over ocean. This land/ocean balance can be interpreted as evidence for global precipitation being constrained by the energy balance in the atmosphere (Allan and Soden, 2007). This balance may also be the explanation for the lack of a trend in the frequency of "high clouds" in the tropical zone reported by Wylie et al. (2005). In the DCCt2 group, which significantly overshoots the tropopause and as such is interesting due to the correlation between overshooting convection and severe storms, the decreasing global trend is not $2 \sigma$ significant. Note that climate change could cause changes not only in DCC frequency but also changes in the spatial relationships between DCCs (i.e., in clustering of DCCs). There is no reason to expect the same trends in the decrease of area occupied by $\omega_{500}<0$ and the DCC occurrence frequency.

\subsection{Extreme events}

Table 2 shows that the tighter DCC selection thresholds identify fewer DCCs, which are more likely associated with microwave overshooting convection, which in turn is presumed to be associated with more severe storms. From Table 3 we also note that the less frequent DCCs (DCCt2 and DCCw4 types) show consistently larger decreases in frequency for ocean and larger increases over land than the less frequent ones (DCCw0 and DCC210 types). We also note that the condition $\omega_{500}<0$ is associated with convection, while DCCs are associated with deep convection. The two quantities are correlated, which may not be too surprising, but the rate of change in the DCCs (convection extremes) for land and ocean is a factor of 2-3 times faster than the change in $\omega_{500}<0$ (mean circulation), as discussed in Sect. 5.2. This finding may fit into a framework of how weather extremes are expected to respond to climate change (cf. Allen and Ingram, 2002; Emori and Brown, 2005).

\section{Summary}

The difference between the brightness temperature measured with AIRS in the $1231 \mathrm{~cm}^{-1}$ atmospheric window channel and the brightness temperature in the strong water vapor 
absorption line at $1419 \mathrm{~cm}^{-1}$ is a measure of the proximity of the cloud tops to the tropopause. This difference and other measures are used to identify DCCs. DCCs referred to a DCCw4 cover $0.16 \%$ of the tropical zone and have cloud tops at or above the tropopause. This is confirmed by simultaneous observations with the AMSU-HSB $183 \mathrm{GHz}$ water vapor channels, which identify about $70 \%$ of the DCCw4 as deep convective, about $40 \%$ as overshooting. The association of DCCs with severe storms has been documented in the literature using infrared, microwave and rain radar studies. In the past $10 \mathrm{yr}$, the frequency of DCCs in the tropical zone has changed insignificantly, but it decreased by about $1 \% \mathrm{yr}^{-1}$ for the tropical ocean, while it increased about $+2 \% \mathrm{yr}^{-1}$ for tropical land. This pattern of essentially zero trend for the tropical zone, but opposite land/ocean trends, is consistent with measurements of precipitation seen in $25 \mathrm{yr}$ of data. However, the $10 \mathrm{yr}$ time span of the AIRS data is too short to observe the reversal of the observed land/ocean trend for DCCs, which is expected based on the precipitation record.

The frequency of DCCs observed over ocean and over land serves as a tracer of tropical circulation patterns. To first order, the changes in the frequency of DCCs are correlated with the change in the fraction of land and ocean areas associated with ascending and descending air motion, defined by vertical speed $\omega$ at $500 \mathrm{hPa}, \omega_{500}$. However, changes in the DCC frequency for land and ocean are a factor of 2-3 times faster than the change in $\omega_{500}$. The correlation of the DCC frequency with the ENSO index and the land/ocean anticorrelation of the trend in the area associated with $\omega_{500}<0$ suggest that the observed changes are part of a decadal variability, which results in small shifts in the tropical circulation pattern, but cancel globally on a 10-year time-scale. The correlation and anti-correlations of the DCC frequency anomaly with MEI and the association of DCCs with storms and heavy precipitation point to an interesting perspective of the regional analysis of past ENSO events, since the SST anomaly in the Nino34 region is available in the form of the extended MEI from 1871. Depending on the selected threshold, the frequency of DCCs in the tropical zone decreases from $0.8 \%$ of $0.06 \%$ area, and the least frequent, more extreme DCCs show the largest trend in frequency, increasing over land, and decreasing over ocean. This finding may fit into the framework of how weather extremes are expected to respond to climate change.

\section{Appendix A}

\section{Anomaly trend evaluation}

We use the time series of the daily fraction of DCCw0 for tropical land to illustrate the anomaly trend analysis procedure. The left panel of Fig. A1 shows the daily DCCw0 frequency. The mean of this time series is 0.0092 with a standard deviation $\sigma=0.0028$, and $5 \%$ of the data exceed the
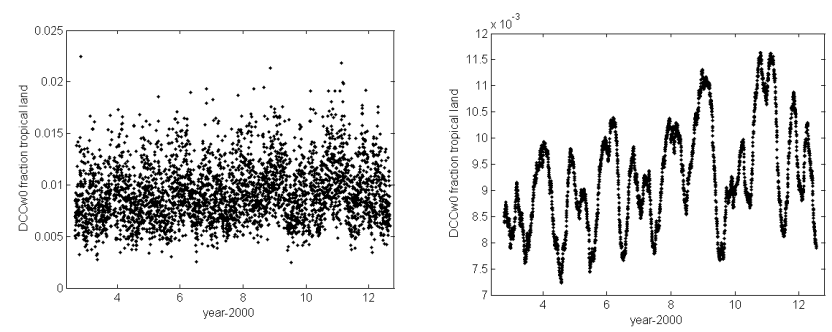

Fig. A1. The left panel shows the day/night averaged daily faction of tropical land associated with DCCw0. The right panel shows the same data, but smoothed with a 96-day running average.

mean $+2 \sigma$. The daily variability is substantial, but the character of the noise appears to be first-order Gaussian. The noise in the daily measurements conceals the annual variability. To illustrate this, the right panel of Fig. A1 shows the same data as the left panel, but the data are smoothed with a 3-month running mean (we use 96 days, because it is a multiple of the EOS Aqua 16-day orbit repeat cycle). The smoothing reveals a pronounced annual variability, which almost dominates over a more complicated pattern starting in 2008. A simple linear fit through the data yields a slope of $+0.00014 \mathrm{yr}^{-1}$ with a $1 \sigma$ slope uncertainty of $0.000022 \mathrm{yr}^{-1}$. The slope is eight times larger than the uncertainty. Expressed as percentage of the mean (0.0092), the trend is $+1.75 \pm 0.22 \% \mathrm{yr}^{-1}$, where the trend uncertainty is $1 \sigma$ confidence.

Visual inspection of Fig. A1 may cast some doubt on the validity of the trend and trend uncertainty derived from the linear analysis. To get a better estimate, we use anomaly trend analysis. For this purpose we fit the data to a low-order harmonic function to $X$, which describes the seasonal variability:

$X_{\text {fit }}=M_{o}+\sum_{n=1 \ldots 6}[a \sin (2 \pi n t)+b \cos (2 \pi n t)]$,

where $t=\left(J-J_{o}\right) / 365.24$ and $J_{o}$ is the Julian day number of the center of the time series. The choice of $n=1, \ldots, 6 \mathrm{ad}-$ equately fits the seasonal variability and is not critical. The anomaly time series $A(t)=\left(x-X_{\text {fit }}\right)$ retains the linear trend of the original time series, and the character of the noise, with the annual and seasonal variability and the mean being removed. By definition $A(t)$ has zero mean. The linear trend of $A(t)$ refers to the anomaly trend. Expressed as percentage of the mean (0.0092), the anomaly trend with $1 \sigma$ confidence is $+1.75 \pm 0.15 \% \mathrm{yr}^{-1}$. In this example the trend did not change. The $1 \sigma$ trend uncertainty decreased significantly compared to the simple linear fit to the data because of the removal of seasonal variability, which contributed to the variance of the original data. 
Acknowledgements. The research described in this paper was carried out at the Jet Propulsion Laboratory, California Institute of Technology, under a contract with the National Aeronautics and Space Administration. We are grateful for the long-term support of Ramesh Kakar, Aqua Program Scientist at NASA HQ.

Edited by: T. J. Dunkerton

\section{References}

Ackerman, S. A.: Global satellite observations of negative brightness temperature differences between 11 and 6.7 microns, J. Atmos. Sci. 53, 2803-2812, 1996.

Adler, R. F. and Fenn, D. D.: Thunderstorm intensity as determined from satellite imagery, J. Appl. Meterrol., 18, 502-517, 1979.

Alcala, C. M. and A. E. Dessler: Observations of deep convection in the tropics using the Tropical Rainfall Measuring Mission (TRMM) precipitation radar, J. Geophys. Res., 107, 4792, doi:10.1029/2002JD002457, 2002.

Allen, M. and Ingram, W. J.: Constraints on future changes in climate and the hydrological cycle, Nature, 419, 224-232, 2002.

Allan, R. P. and Soden, B. J.: Large discrepancy between observed and simulated precipitation trends in the ascending and descending branches of the tropical circulation, Geophys. Res. Lett., 34, L18705, doi:10.1029/2007GL031460, 2007.

Aumann, H. H., Broberg, S., Elliott, D., Gaiser, S., and Gregorich, D.: Three years of Atmospheric Infrared Sounder radiometric calibration validation using sea surface temperatures, J. Geophys. Res., 111, D16S90, doi:10.1029/2005JD006822, 2006.

Aumann, H. H., DeSouza-Machado, S. G., and Behrangi, A.: Deep convective clouds at the tropopause, Atmos. Chem. Phys., 11, 1167-1176, doi:10.5194/acp-11-1167-2011, 2011.

Aumann, H. H., Elliott, D., and Strow, L. L.: Validation of the Radiometric Stability of the Atmospheric Infrared Sounder, Proc. SPIE Optics and Photonics Conference, San Diego, August 2012, Session 8510, Earth Observing System XVII, edited by: Buttler, J., Xiong, X., and Gu, X., 2012.

Bedka, K., Brunner, J., Dworak, R., Feltz, W., Otkin, J., and Greenwald, T.: Objective Satellite-based Overshooting Top Detection Using Infrared Window Channel Brightness Temperature Gradients, J. Appl. Meteor. Climatol., 49, 181-202,, doi:10.1175/2009JAMC2286.1, 2010.

Bony, S., Dufrense, J.-L., Le Treut, H., Morcrette, J.-J., and Senior, C.: On dynamic and thermodynamic components of cloud changes, Clim. Dynam., 22, 71-86, doi:10.1007/s00382-0030369-6, 2004.

Emori, S. and Brown, S. J.: Dynamic and thermodynamic change in mean and extreme precipitation under changed climate, Geophys. Res. Lett., 32, L17706, doi:10.1029/2005GL023272, 2005.

Fueglistaler, S., Dessler, A. E., Dunkerton, T. J., Folkins, I., Fu, Q., and Mote, P. W.: Tropical Tropopause Layer, Rev. Geophys., 47, RG1004, doi:10.1029/2008RG000267, 2009.

Gettelman, A., Salby, M. L., and Sassi, F.: Distribution and influence of convection in the tropical tropopause region, J. Geophys. Res., 107, doi:10.1029/2001JD001048, 2002.

Gettelman, A., Birner, T., Eyring, V., Akiyoshi, H., Bekki, S., Brühl, C., Dameris, M., Kinnison, D. E., Lefevre, F., Lott, F., Mancini, E., Pitari, G., Plummer, D. A., Rozanov, E., Shibata, K., Stenke, A., Struthers, H., and Tian, W.: The Tropical
Tropopause Layer 1960-2100, Atmos. Chem. Phys., 9, 16211637, doi:10.5194/acp-9-1621-2009, 2009.

Hong, G., Heygster, G., Miao, J., and Kunzi, K.: Detection of tropical deep convective clouds from AMSU-B water vapor channels measurements, J. Geophys. Res., 110, D05205, doi:10.1029/2004JD004949, 2005.

Janowiak, J. E. and Arkin, P. A.: Rainfall variations in the tropics during 1986-1989, as estimated from observations of cloudtop temperatures, J. Geophys. Res., 96, Supplement, 3359-3373, 1991.

Joyce, R. and Arkin, P. A.: Improved estimates of tropical and subtropical precipitation using the GOES Precipitation Index, J. Atmos. Ocean. Technol., 10, 997-1011, 1997.

Kalney, E. et al.: The NCEP/NCAR 40 year reanalysis project, B. Amer. Meteor. Soc., 77, 437-471, 1996.

Lambrigtsen, B. H. and Calheiros, R. V.: The Humidity Sounder for Brazil - An international partnership, IEEE Trans. Geosci. Remote Sens., TGARS, 41, 352-361, 2003.

Lambrigtsen, B. and Lee, S. Y.: Coalignment and Synchronization of the AIRS Instrument Suite, IEEE Trans. Geosci. Remote Sens., 41, 343-351, 2003.

Liu, C. and Zipser, E. J.: Global distribution of convection penetrating the tropical tropopause, J. Geophys. Res., 110, D23104, doi:10.1029/2005JD006063, 2005.

Liu, C. and Zipser, E. J.: Diurnal cycles of precipitation, clouds and lightning in the tropics from 9 years of TRMM observations, Geophys. Res. Lett., 35, L04819, doi:10.1029/2007GL032437, 2008.

Liu C., Zipser, E. J., and Nesbitt, S.: Global Distribution of Tropical Deep Convection: Different Perspectives from TRMM Infrared and Radar Data, J. Climate, 20, 489-503, 2007.

Mapes, B. and Houze, R.: Coud Clusters and Superclusters over the Oceanic Warm Pool, Mon. Weather Rev., 121, 1898-1415, 1993.

Reynolds, D. W.: Observations of Damaging Hailstorms from Geosynchronous Satellite Digital Data, Mon. Weather Rev., 108, 337-348, 1980.

Schmetz, J., Tjemkes, S. A., Gube, M., and Van De Berg, L.: Monitoring deep convection and convective overshooting with METEOSAT, Adv. Space Res., 433-441, 1997.

Setvak, M., Lindsey, D. T., Rabin, R. M., Wang, P. K., and Demeterova, A.: Indication of water vapor transport into the lower stratosphere above midlatitude convective storms: Meteosat Second Generation satellite observations and radiative transfer simulations, J. Atmos. Res., 89, 170-180, 2008.

Setvak, M., Bedka, K., Lindsey, D., Sokol, A., Charvat, Z., Staska, P., and Wang, P.: A-Train observations of deep convective storm tops, J. Atmos. Res., doi:10.1016/2012.06.020, in press, 2012.

Sherwood S. and Dessler, A.: On the control of stratospheric humidity, Geophys. Res. Lett., 27, 677-680, 2000.

WCRP report on Global Precipitation, WCRP-128, WMP/TDNo.1430, 2008.

Webster, P. J.: Large-scale structure of the tropical atmosphere, edited by: Hoskins, B. J. and Pearce, R. P., General Circulation of the Atmosphere, London, Academic Press, 235-275, 1983.

Wolter, K. and Timlin, M. S.: Measuring the strength of ENSO events - how does 1997/98 rank?, Weather, 53, 315-324, 1998.

Wolter, K. and Timlin, M. S.: El Niño/Southern Oscillation behaviour since 1871 as diagnosed in an extended multivari- 
ate ENSO index (MEI.ext), Intl. J. Climatol., 31, 1074-1087, doi:10.1002/joc.2336, 2011.

Wylie, D., Jackson, D. J., Menzel, W. P., and Bates, J. J.: Trends in high and low clouds from the analysis of 20 years of HIRS and ISCCP data, J. Climate, 18, 3021-3031, 2005.
Young, A., Bates J. J., and Curry, J. A.: Complementary use of passive and active remote sensing for the detection of penetrating convection from CloudSat, Calypso and Aqua MODIS, J. Geophys. Res., 117, D13205, doi:10.1029/2011JD016749, 2012. 